\title{
Additive versus Multiplicative Seasonality in Solar Radiation Time Series
}

\author{
John Boland ${ }^{a}$ \\ ${ }^{a}$ School of ITMS, University of South Australia, Mawson Lakes, South Australia, 5095 \\ Email: john.boland@unisa.edu.au
}

\begin{abstract}
Several approaches have been developed for the forecasting of solar radiation on various time scales. The approaches range from use of Artificial Neural Networks (ANN) using raw solar irradiance to several methods utlizing first some type of seasonal adjustment. This seasonal adjustment is either in the form of multiplicative deseasoning such as using clearness index or a clear sky model, or additive deseasoning using Fourier series or wavelets. This article focuses on comparison of various methods of seasonal adjustment, but first let us examine the range of forecasting tools subsequent to this process of the modelling. The tools range from ANN to Adaptive Autoregressive to Exponential Smoothing. As well as these single method approaches, several authors utilise what might be called hybrid models, like wavelets plus ANN, and the Coupled Autoregressive and Dynamical Systems (CARDS) model of the present author and colleagues. This is not an exhaustive list but it gives the idea of the wide range of possible methods used for short term forecasting of solar radiation. There are essentially two methods of dealing with the seasonalities inherent in climate variables. One can deal with it in a multiplicative or additive modelling framework. Interestingly, though The multiplicative approach is problematic, it is the approach most often used for solar forecasting models in particular. There are two versions of the multiplicative approach with respect to solar radiation, calculating the clearness index, and estimating the clear sky index. There is this distinction in terminology since to form the clearness index, one divides the global solar radiation by the extraterrestrial radiation, a quantity determined only via astronomical formulae. On the other hand the clear sky index involves dividing the global radiation by a clear sky model. Note that the wind resource is not as seasonally dependent as the solar radiation, and both multiplicative and additive versions of dealing with seasonality are used. Additive de-seasoning is enacted through subtracting a mean function from the solar radiation, that function formed usually through the addition of terms involving a basis of the function space. Arguments are presented to support the conjecture that the appropriate way to perform this operation is through the use of a Fourier set of basis functions. As well as specifically dealing with how appropriate this approach is for solar radiation, it will discussed with reference to modelling ambient temperature. There are occasions when the bivariate modelling of temperature and solar radiation is needed. For example, a solar photovoltaic cell loses efficiency as the temperature increases, and so for evaluating the expected performance of a PV cell, it would be useful to be able to model temperature and solar radiation together. It is not possible to deal with temperature seasonality via a multiplicative approach. It would be difficult to construct a bivariate time series model where one dealt with the seasonality with a multiplicative approach with one variable and an additive with the other. This is another compelling argument for dealing with the solar radiation seasonality using Fourier series.
\end{abstract}

Keywords: Time series, Additive seasonality, multiplicative seasonality, Fourier Series, Clear Sky Index 


\section{INTRODUCTION}

Several approaches have been developed for the forecasting of solar radiation on various time scales. A comprehensive review of recent articles can be seen in (Inman et al., 2013), (Diagne et al., 2013). The approaches range from use of Artificial Neural Networks (ANN) using raw solar irradiance (Sfetsos and Coonick, 2000) to several methods utlizing first some type of seasonal adjustment. This seasonal adjustment is either in the form of multiplicative deseasoning such as using clearness index or a clear sky model, or additive deseasoning using Fourier series or wavelets. I shall comment on these various methods of seasonal adjustment, but first let us examine the range of forecasting tools subsequent to this process of the modelling. The tools range from ANN (Kemmoku et al., 1999), (González-Romera et al., 2008), (Sfetsos and Coonick, 2000) and several other references, to Adaptive Autoregressive (Bacher et al., 2009) to Exponential Smoothing (Dong et al., 2013). As well as these single method approaches, several authors utilise what might be called hybrid models, like wavelets plus ANN (Cao and Cao, 2006), (Cao and Lin, 2008), (Mellit et al., 2006), and the Coupled Autoregressive and Dynamical Systems (CARDS) model of the present author and colleagues (Huang et al., 2013). This is not an exhaustive list but it gives the idea of the wide range of possible methods used for short term forecasting of solar radiation. I have not even delved into the more recent array of approaches utilising sky cameras and so forth.

\section{SEASONALITY}

I would now like to discuss in more detail the methods referred to above of dealing with the inherent seasonality in sub-daily solar radiation series, since the focus of this paper is forecasting on hourly and sub-hourly time scales. As mentioned, one approach has been to use multiplicative deseasoning in the form of dividing the solar radiation series by, in some cases, the clearness index (Martín et al., 2010), (Aguiar and Collares-Pereira, 1992), (Cena et al., 1979), (Skartveit and Olseth, 1992). Alternatively, numerous articles deal with dividing the solar radiation by some clear sky model to create a clear sky index (Bacher et al., 2009), (Kemmoku et al., 1999). It is useful to examine what type of situation is usually handled by implementing a multiplicative seasonal model. The multiplicative model is more prevalent with economic series since most seasonal economic series have seasonal variation which increases with the level of the series. One example is in the time series of tourist arrivals (Lim and Mcaleer, 2001). Interestingly, they deal with the seasonality by taking logarithms of the data. In fact, one researcher has done similar with solar data (Reikard, 2009). One could make an argument for using multiplicative deseasoning for solar data by using division with clearness index or a clear sky model as well, since the seasonality has a varying amplitude over the year. Note that, as will be shown below, using an additive Fourier series representation of the seasonality deals with this more effectively. If one does use multiplicative deseasoning, one could make a case for the use of the clearness index rather than a clear sky model, even though Paoli et al. (2010) discusses the use of both methods and goes for a clear sky index. Since Ineichen (2006) feels it is necessary to examine the relative efficacy of numerous clear sky models, this points to using the clearness index being a better approach. This is because the clearness index approach utilises a variable calculated through astronomical formulae, rather than being the result of a model, with a number of inherent assumptions.

There are compelling arguments as to why an additive seasonal modelling process is preferable to a multiplicative one. This is especially true in the case of a Fourier series model. There are two ways one can think of this approach - in a statistical sense, if sufficient years are used for the parameter estimation, the expected value for each time period is given. Also, there are simple formulae for calculating the amount of variance in the data explained at each chosen frequency. In a physical sense, the Fourier series model gives the climate for the location. In a very practical sense, the whole process is data driven, distinct from the clear sky index method. A key point, is as Skeiker (2006) has reflected, that there is a richness of physical meaning inherent in this representation which is not necessarily apparent in other methods. Dong et al. (2013) identify a number of significant sub-diurnal cycles inherent in the solar radiation time series, but unfortunately does not go into the physical meaning of them. Boland (2008) delves into the physical nature of the radiation series as exemplified by the significant frequencies embedded within the data, but one particular characteristic stands out. The daily cycle is obviously a significant component. The twice daily cycle, as also identified by Dong et al. (2013) is too, but the most interesting physical aspect is the necessity of including the frequencies just surrounding those two, at 364, 365 and 729, 731 cycles per year, the so-called sidebands or beat frequencies - see Figure 1 . This example is for the town of Mildura, Australia, latitude $-34.22^{\circ}$. So the Fourier series contains 11 significant frequencies: 


$$
\begin{aligned}
S_{t}= & \alpha_{0}+\alpha_{1} \cdot \cos \frac{2 \pi t}{8760}+\beta_{1} \cdot \sin \frac{2 \pi t}{8760}+\alpha_{2} \cdot \cos \frac{4 \pi t}{8760}+\beta_{2} \cdot \sin \frac{4 \pi t}{8760}+ \\
& \sum_{n=1}^{2} \sum_{m=-1}^{1}\left(\alpha_{n m} \cdot \cos \frac{2 \pi(356 n+m) t}{8760}+\beta_{n m} \cdot \sin \frac{2 \pi(365 n+m) t}{8760}\right)
\end{aligned}
$$

In discussing the question of the multiplicative modelling of solar radiation, it was suggested that one of the reasons why one might choose it was the change of amplitude with level of the series. The most significant aspect of this with solar radiation series, and it should be noted, with other climate data series, is the varying amplitude of the daily cycle as one progresses through the year. The amplitude is higher in summer than winter but the progression is systematic over the year. The inclusion of the beat frequencies in the Fourier series specifically captures this amplitude modulation, and this is a compelling reason for the representation of the series via this method. See Figures 2,3 for the effect of ignoring the sidebands. Figure 4 gives a vivid

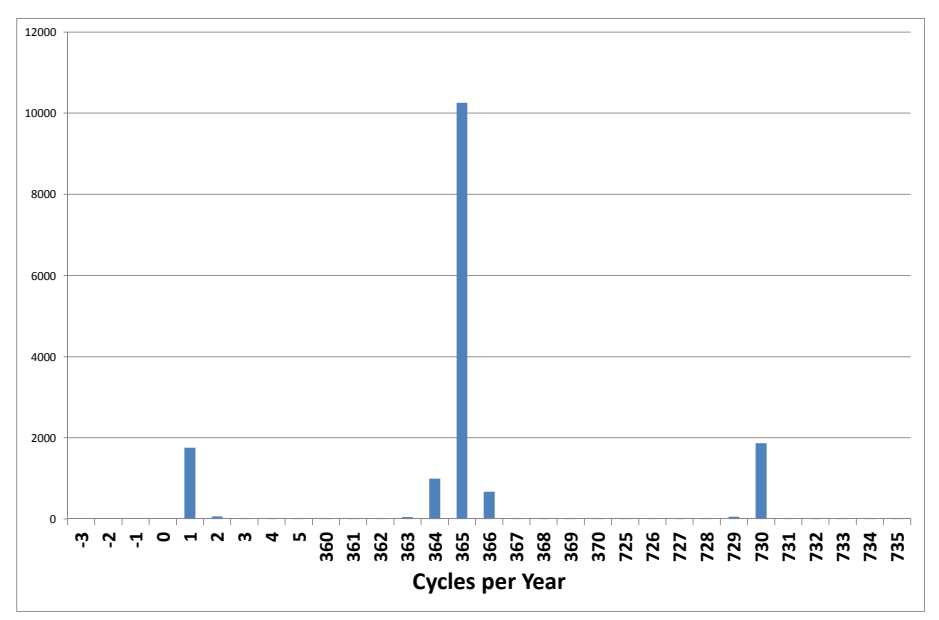

Figure 1. Power spectrum for hourly solar radiation for Mildura data

illustration of the need for the inclusion of the amplitude modulating frequencies. It shows what the actual amplitude over the day is for each day of the year, plus what the Fourier series representation gives without the contribution from the sideband frequencies. Figure 5 gives the amplitude with the sidebands in. Inclusion reduces the amplitude in winter and increases it in summer to ensure zero (with minor errors due to using a small number of frequencies) radiation at night. Figure 6 is the same as Figures 2, 3 but with the contributions from the sidebands frequencies. I would suggest that this depiction amply illustrates that the intended benefit from using multiplicative deseasoning has been more than amply catered for, and in an internally consistent manner with additive deseasoning through the use of Fourier series. It is internally consistent in that the physical interpretation of each term that is included is inherently simple and demonstrable.

\subsection{CORRESPONDENCE WITH OTHER CLIMATE VARIABLES}

The final reason I would put forward for the use of Fourier series, or at least an additive model of seasonality is the correspondence with other climate variables. As Skeiker (2006) points out, it is a good representation for temperature seasonality as well. One might argue that there is no necessity to treat all variables with the same methods, but there is a very good reason for using the same methods for solar and temperature at least. This is that when modelling the performance of crystalline solar cells for instance, one must construct a bi-variate model for the two variables since the performance or the cells is dependent on temperature as well as solar, and temperature has a lagged dependence on solar. This lagged dependence can only be adequately modelled when one first finds all serial dependence for each series independently, and then investigates the cross correlation between white noise series. This is most sensibly done if one uses the same modelling structure for each. Given that there is no temperature equivalent of a clear sky model, the way this can be effected is via using Fourier series to model the seasonality. 
J. Boland, Additive versus Multiplicative Seasonality in Solar Radiation Time Series

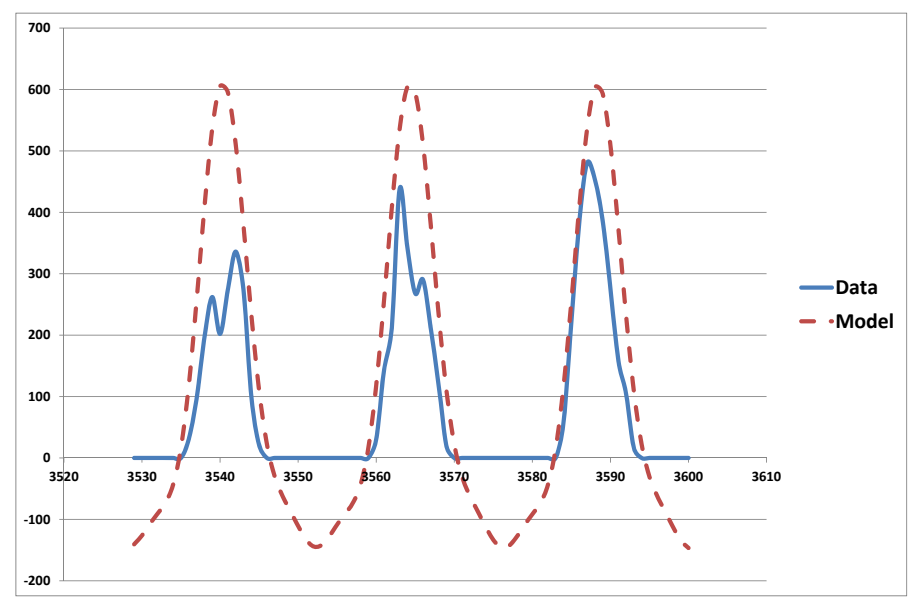

Figure 2. Effect of no sidebands on winter solar radiation in Watts, for three days of hourly data

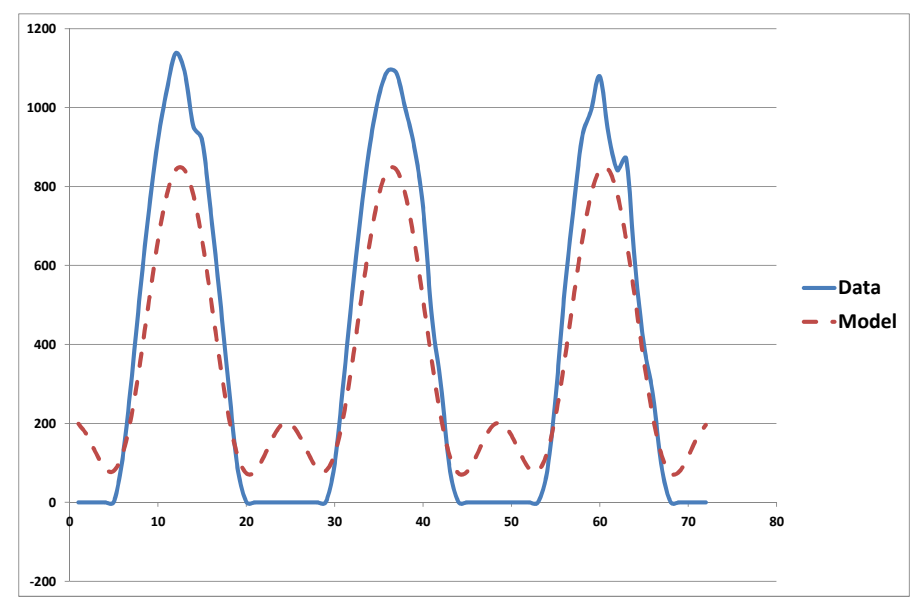

Figure 3. Effect of no sidebands on summer solar radiation in Watts, for three days of hourly data

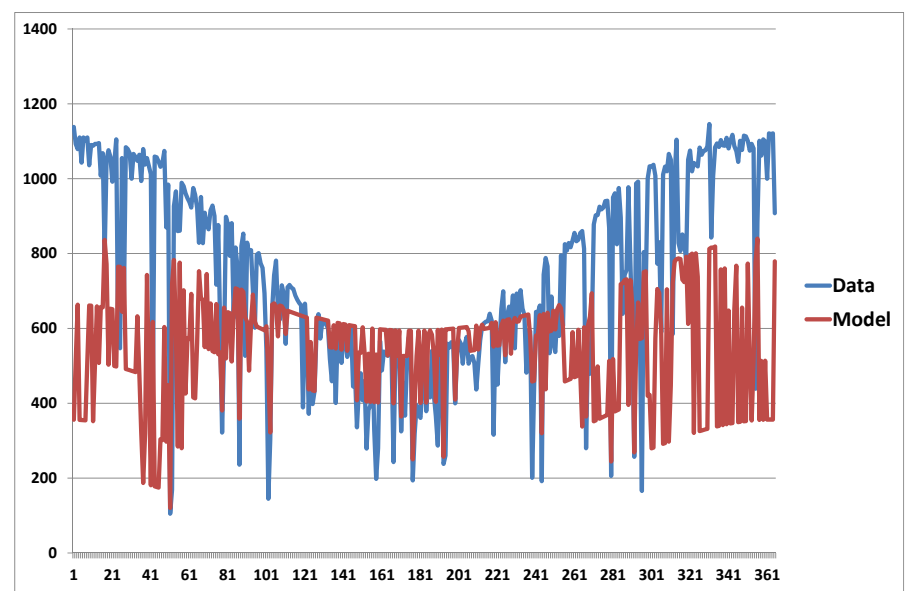

Figure 4. Daily amplitude with no sidebands, in Watts, over the year

\section{CLEAR SKY MODELS}

The clear sky index (CSI) is formed by dividing the solar irradiance by a suitable clear sky model - as in Equation 2. As stated previously, there are numerous clear sky models (Ineichen (2006)). 


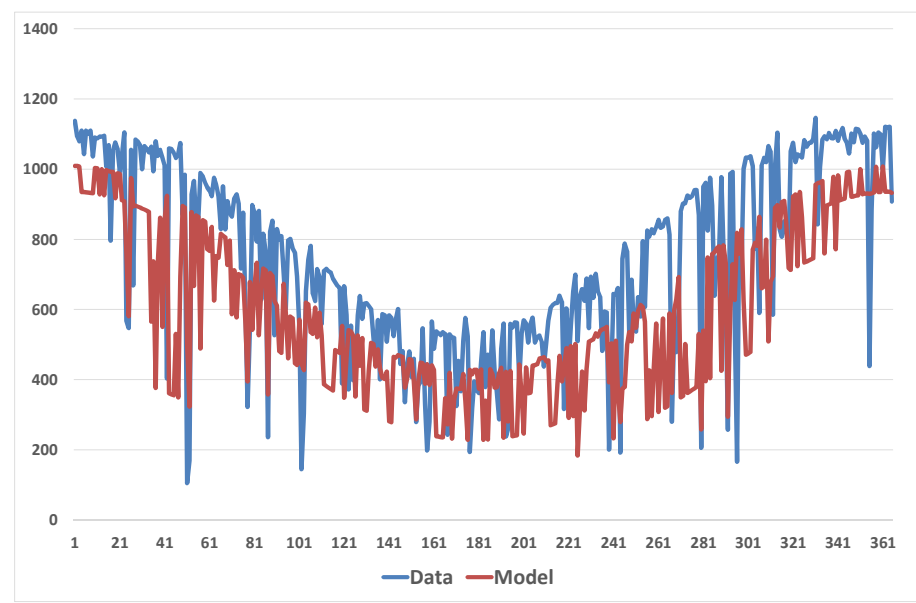

Figure 5. Daily amplitude with sidebands, in Watts, over the year

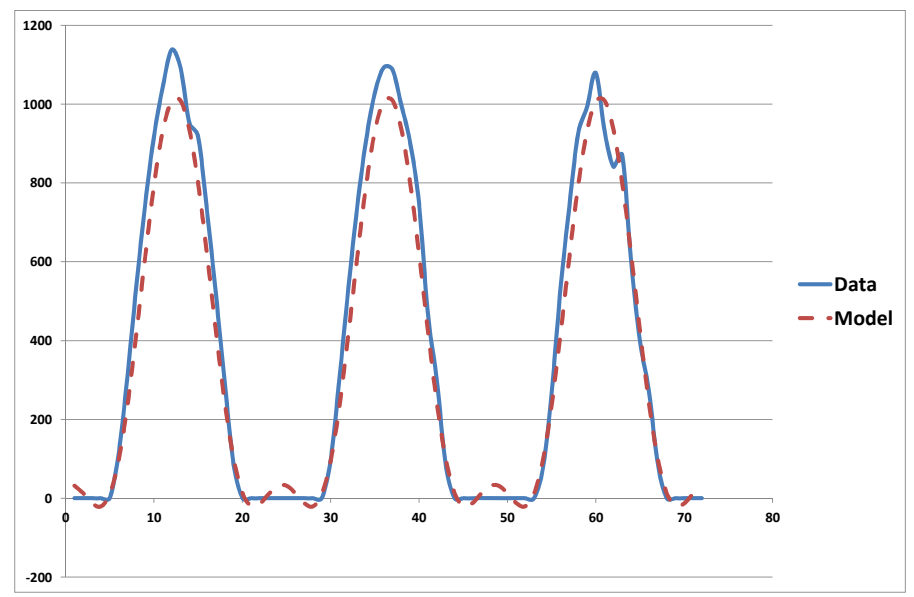

Figure 6. Three days of hourly data plus model with sidebands included

$$
k^{*}=\frac{I}{I_{\text {Clear }}}
$$

Here $k^{*}$ is the Clear Sky Index (CSI), $I$ is the solar radiation at the Earth's surface, and $I_{\text {Clear }}$ is the clear sky model value for the same interval. The expectation with the CSI is that it will fluctuate with values mostly less than unity. It is of course possible, through extra cloud reflection in particular, to have some values greater than one. However, when one examines the data formed when the CSI is calculated, two problems emerge. It is often the case that values close to sunrise and sunset are greater than one. This is especially the case with hourly data since sunrise or sunset may will occur within an hour, not on the boundary. Even if you restrict the data to times for which the solar altitude $\alpha>10^{\circ}$, as is common, this problem can persist. The other problem occurs when you have a location with significantly clearer skies than the model has been designed to cater for. In this case I suggest it is like the mathematical theory paradigm. You only need one counterexample to disprove a conjecture. I will use CSI values for Los Vegas hourly data for 2010 to illustrate the two problems. The former is shown in Figure 7, with significant numbers of values greater than one for both cases of daylight hours and hours with $\alpha>10^{\circ}$. The latter is shown in Figure 8, where even for $\alpha>10^{\circ}$, the values at the beginning and end are inflated. 


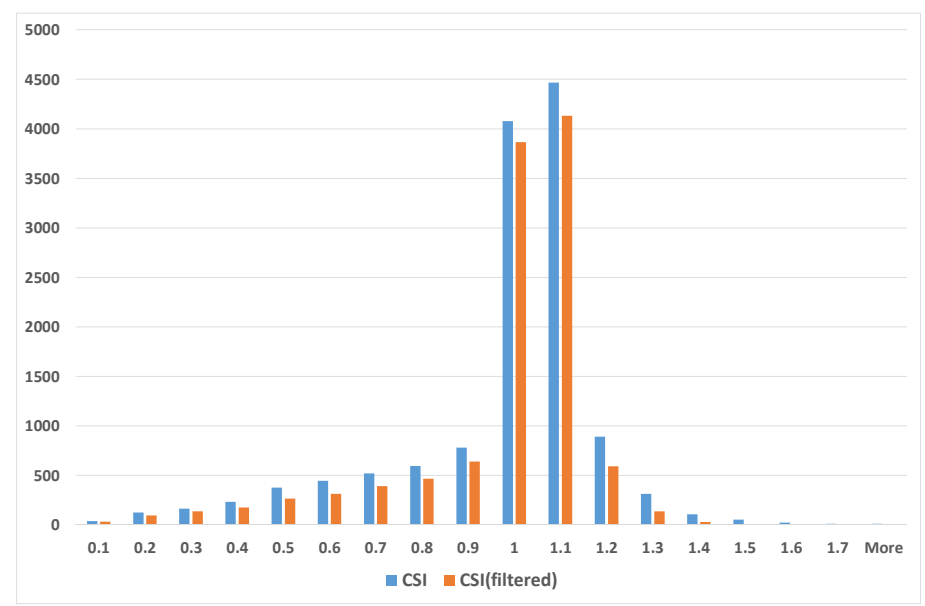

Figure 7. Histogram of CSI values for Los Vegas

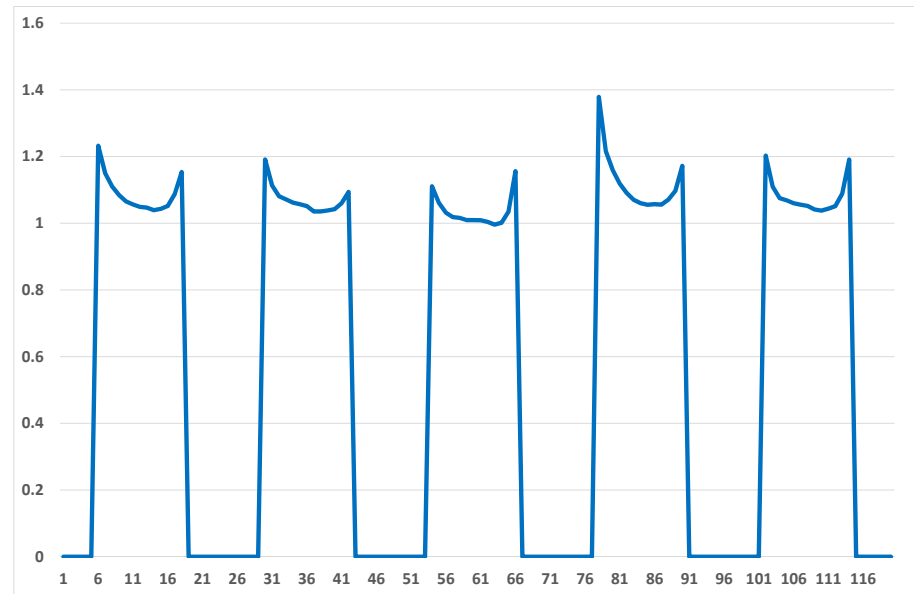

Figure 8. Five days of CSI for Los Vegas

\section{CONCLUSIONS}

The paper compares the description of seasonality and its removal through two mechanisms, as applied to hourly solar radiation time series. A significant number of researchers use multiplicative deseasoning, through division of the data by a selected clear sky model for radiation. I suggest that additive deseasoning through subtraction of an appropriate Fourier series model, is more suitable. Perhaps the original proponents of the clear sky index approach were drawn to it by the fact of zero radiation at night, and so thought this approach was more reasonable. I have provided arguments why it presents problems in many instances. However, I also argue that the use of Fourier series reveals more about the physical nature of the phenomenon and thus is the more useful approach.

\section{REFERENCES}

Aguiar, R. and M. Collares-Pereira (1992). TAG: a time dependent, autoregressive, gaussian model for generating synthetic hourly radiation. Solar Energy (49), 167-174.

Bacher, P., H. Madsen, and H. A. Nielsen (2009, October). Online short-term solar power forecasting. Solar Energy 83(10), 1772-1783.

Boland, J. (2008). Time series and statistical modelling of solar radiation. Recent Advances in Solar Radiation Modelling Chapter 11, 283-312. 
J. Boland, Additive versus Multiplicative Seasonality in Solar Radiation Time Series

Cao, J. and S. Cao (2006, December). Study of forecasting solar irradiance using neural networks with preprocessing sample data by wavelet analysis. Energy 31(15), 3435-3445.

Cao, J. and X. Lin (2008, June). Study of hourly and daily solar irradiation forecast using diagonal recurrent wavelet neural networks. Energy Conversion and Management 49(6), 1396-1406.

Cena, V., C. Mustacchi, and M. Rocchi (1979). Stochastic simulation of hourly global radiation sequences. Energy $23,47-51$.

Diagne, M., M. David, P. Lauret, J. Boland, and N. Schmutz (2013, November). Review of solar irradiance forecasting methods and a proposition for small-scale insular grids. Renewable and Sustainable Energy Reviews 27, 65-76.

Dong, Z., D. Yang, T. Reindl, and W. M. Walsh (2013, June). Short-term solar irradiance forecasting using exponential smoothing state space model. Energy 55, 1104-1113.

González-Romera, E., M. Jaramillo-Morán, and D. Carmona-Fernández (2008, November). Monthly electric energy demand forecasting with neural networks and Fourier series. Energy Conversion and Management 49(11), 3135-3142.

Huang, J., M. Korolkiewicz, M. Agrawal, and J. Boland (2013, January). Forecasting solar radiation on an hourly time scale using a Coupled AutoRegressive and Dynamical System (CARDS) model. Solar Energy 87, 136-149.

Ineichen, P. (2006, April). Comparison of eight clear sky broadband models against 16 independent data banks. Solar Energy 80(4), 468-478.

Inman, R. H., H. T. Pedro, and C. F. Coimbra (2013, December). Solar forecasting methods for renewable energy integration. Progress in Energy and Combustion Science 39(6), 535-576.

Kemmoku, Y., S. Orita, S. Nakagawa, and T. Sakakibara (1999). Daily insolation forecasting using a multistage neural network. 66(3), 193-199.

Lim, C. and M. Mcaleer (2001). Forecasting tourist arrivals. 28(4), 965-977.

Martín, L., L. F. Zarzalejo, J. Polo, A. Navarro, R. Marchante, and M. Cony (2010, October). Prediction of global solar irradiance based on time series analysis: Application to solar thermal power plants energy production planning. Solar Energy 84(10), 1772-1781.

Mellit, a., M. Benghanem, and S. Kalogirou (2006, July). An adaptive wavelet-network model for forecasting daily total solar-radiation. Applied Energy 83(7), 705-722.

Paoli, C., C. Voyant, M. Muselli, and M.-L. Nivet (2010, December). Forecasting of preprocessed daily solar radiation time series using neural networks. Solar Energy 84(12), 2146-2160.

Reikard, G. (2009, March). Predicting solar radiation at high resolutions: A comparison of time series forecasts. Solar Energy 83(3), 342-349.

Sfetsos, A. and A. H. Coonick (2000). Univariate and multivariate forecasting of hourly solar. Solar Energy 68(2), 169-178.

Skartveit, A. and J. Olseth (1992). The probability density and autocorrelation of short-term global and beam irradiance. Solar Energy 49, 477-487.

Skeiker, K. (2006, July). Mathematical representation of a few chosen weather parameters of the capital zone Damascus in Syria. Renewable Energy 31(9), 1431-1453. 